\title{
VARIABLES RELACIONADAS CON EL NÚMERO Y ELECCIÓN DE LAS REDES SOCIALES DE LOS HOTELES ANDALUCES DE 4 Y 5 ESTRELLAS
}

\author{
Inmaculada Rabadán-Martín* \\ Universidad de Huelva \\ https://orcid.org/0000-0001-5192-6928 \\ Nuria Padilla-Garrido \\ Universidad de Huelva \\ https://orcid.org/0000-0002-2988-2663 \\ Francisco Aguado-Correa \\ Universidad de Huelva \\ https://orcid.org/0000-0001-8306-2917
}

\section{RESUMEN}

La gestión de las redes sociales se ha convertido en un factor clave para el turismo. Los contenidos generados en las mismas pueden condicionar la elección por parte de los turistas, la reputación online, las ventas, e incluso la supervivencia de un establecimiento hotelero. A través del estudio de 503 hoteles andaluces de 4 y 5 estrellas, nuestro trabajo analiza la totalidad de redes sociales presentes en sus páginas web oficiales, así como los posibles factores (número de estrellas, modalidad, antigüedad, tamaño y pertenencia a cadena) que pueden influir en el número y la elección de estas.

Palabras clave: redes sociales; hoteles; páginas web; turismo 2.0.

Variables relating to the number and the choice of social networks among four and five-star Andalusian hotels

Fecha de recepción: 27 de febrero de 2019

Fecha de aceptación: 24 de julio de 2019

* Facultad de Ciencias Empresariales y Turismo. Universidad de Huelva. Plaza de la Merced, $\mathrm{n}^{\circ} 11.21071$ HUELVA (España).E-mail: rabadan@uhu.es, padilla@uhu.es, agucor@uhu.es 


\begin{abstract}
Social network management has become a key factor in developing tourism. Content generated on social networks can influence tourist choices, online reputation, sales and the very survival of the hotel establishment.

In a study of 503 four- and five-star hotels in Andalusia, we analysed all the social networks used on their official websites to attract customers, and the potential factors (number of stars, modality, age of the establishment, size, chain hotel or otherwise) that could influence the number of visits and choice of destination.
\end{abstract}

Keywords: social networks; hotels; web pages; tourism 2.0.

\title{
1. INTRODUCCIÓN
}

En los últimos años, las redes sociales se han convertido en un factor clave para el turismo. Según el Barómetro de TripAdvisor 2016 (TripAdvisor, 2016), ante la pregunta del uso de las redes sociales para planificar su alojamiento, el 39\% de los 36.444 viajeros encuestados en 33 países afirmó basarse en la experiencia de otros viajeros, el $16 \%$ en el contenido publicado por los propios establecimientos y el $12 \%$ en la opinión de familiares y amigos. La importancia de las opiniones online también queda resaltada en el TripBarometer 2017/2018 (TripAdvisor, 2018), en el que se indica que el 86\% de los viajeros no reservaría un alojamiento sin haber leído opiniones sobre el mismo previamente.

Como reflejo de la amplia accesibilidad y utilización de estas redes, los consumidores han cambiado la forma de analizar destinos, hacer reservas, aprender sobre nuevas ofertas, planificar itinerarios y comunicarse con proveedores (Phelan et al., 2013). En cada etapa del viaje, los turistas utilizan diferentes tipos de redes sociales (Verma et al., 2012), sin embargo, su papel más relevante tiene lugar en la etapa previa, cuando consultan medios para la planificación, la búsqueda de información y la toma de decisiones (Cox et al., 2009; Zeng, 2013). En esta fase, los contenidos generados por otros usuarios constituyen una pieza clave a la hora de adoptar una decisión final, así como para minimizar los riesgos percibidos (Fotis et al., 2012; Sigala et al., 2012; Leung et al., 2013) en un sector en el que suele desconocerse la calidad del producto o servicio antes del consumo.

Desde el punto de vista empresarial, este fenómeno también ha tenido importantes implicaciones. Tradicionalmente, las empresas han controlado la información online debido al carácter pasivo de los consumidores (Ayeh et al., 2012). Sin embargo, hoy en día las redes sociales han cobrado tal protagonismo que pueden determinar las estrategias de marketing, la reputación, las ventas e incluso la supervivencia de una empresa (Kietzmann et al., 2011). Por todo ello, los investigadores han abogado por su utilización a nivel empresarial (Zehrer et al., 2011), destacando los beneficios que pueden aportar (SánchezFranco y Rondan-Cataluña, 2010). Sin embargo, hay estudios que también evidencian la reticencia o la incapacidad de muchos profesionales para utilizarlas como herramienta 
estratégica (Kietzmann et al., 2011). Por todo ello, es necesario saber hasta qué punto están implementadas en la práctica.

En este sentido, y en lo referente al sector hotelero español, las investigaciones se han centrado, básicamente, en el estudio de la utilización y gestión de las redes sociales en establecimientos de tres estrellas o más, pertenecientes o no a cadenas, ubicados, principalmente, en la costa de distintas regiones (Martínez et al., 2012 y 2013; Cañero et al., 2015; Cascales et al., 2017; Mellinas et al., 2014 y 2016). Dichos estudios han revelado que la mayor parte de los hoteles las aprovechan como herramienta de comunicación, utilizándolas preferentemente los establecimientos pertenecientes a cadenas como canal de ventas. También han evidenciado que su uso adecuado está relacionado con una gestión eficiente, así como con una mayor nota en Booking o TripAdvisor. Por otra parte, los hoteles con mayor número de estrellas tienden a utilizarlas en mayor proporción, destacando especialmente Facebook y Twitter.

A pesar del avance que suponen estos estudios, reducen el análisis de las redes sociales a cuatro o cinco como máximo. En la mayoría de los casos, no realizan un análisis estadístico que permita contrastar la representatividad de las posibles relaciones entre las variables estudiadas. Además, olvidan aspectos tan interesantes en el estudio de las redes como la modalidad hotelera, la fecha de inicio de la actividad o el tamaño del establecimiento.

Por todo ello, nuestro trabajo tiene como objetivo analizar la totalidad de redes sociales presentes en las páginas web de los hoteles andaluces de 4 y 5 estrellas, así como los posibles factores (número de estrellas, modalidad, antigüedad, tamaño y pertenencia a cadena) que pueden estar relacionadas con el número y la elección de las mismas, comprobando, mediante un contraste de hipótesis, el nivel de significación de las relaciones entre las variables analizadas. Para ello, una vez descrito el mapa del sector hotelero andaluz y delimitadas las redes sociales a estudiar, presentaremos la metodología del trabajo, los resultados obtenidos y las conclusiones del mismo.

\section{MAPA DEL SECTOR HOTELERO ANDALUZ}

Según el "Balance del año turístico en Andalucía 2017" (Consejería de Turismo y Deporte, 2018), en 2017 los ingresos globales por turismo en esta comunidad alcanzaron el $12,8 \%$ de su PIB. Además, la demanda de bienes y servicios de los turistas provocó una serie de efectos directos e indirectos en la economía de esta región, que se estimaron en 25,8 miles de millones de euros, lo cual evidencia la importancia de este sector para Andalucía.

Centrándonos en la industria hotelera andaluza, el Gráfico 1 nos permite contextualizar el mapa por provincias en el año 2017, pudiéndose observar que, por número de estrellas y por orden, los hoteles de 2, 4 y 3 estrellas fueron los más numerosos. De acuerdo con el número de plazas ofertadas, Málaga lideraba la clasificación, seguida, con gran diferencia, por Cádiz y Granada. Los hoteles que disponían de un mayor número de plazas en toda Andalucía fueron los de 4 estrellas (Gráfico 2). En relación con el tamaño medio de los hoteles, las provincias de Jaén y Córdoba se caracterizaban por tener una proporción menor entre plazas y establecimientos hoteleros, lo cual indica que los hoteles tenían un tamaño medio inferior. 


\section{Gráfico 1}

HOTELES POR NÚMERO DE ESTRELLAS, PROVINCIA Y TOTAL DE PLAZAS OFERTADAS EN 2017 EN TODA ANDALUCÍA

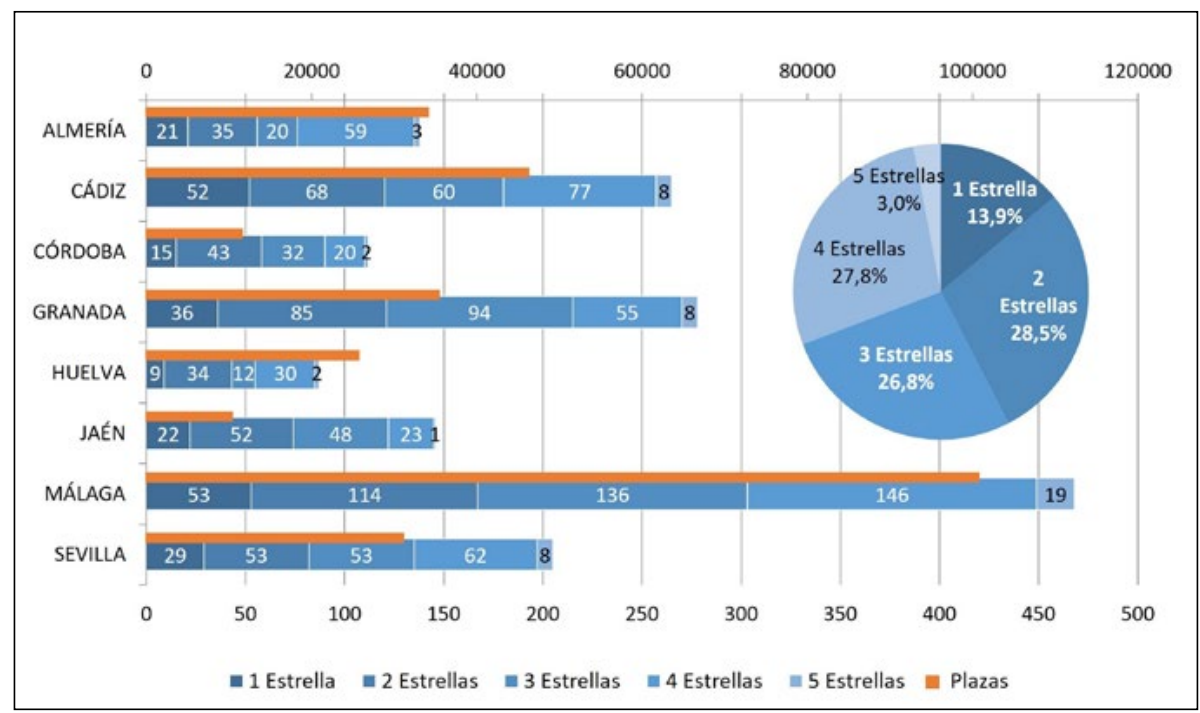

Fuente: Elaboración propia a partir de Consejería de Turismo y Deporte, 2017b.

\section{Gráfico 2}

\section{DISTRIBUCIÓN PORCENTUAL DE LAS PLAZAS HOTELERAS SEGÚN} NÚMERO DE ESTRELLAS Y PROVINCIA (2017)

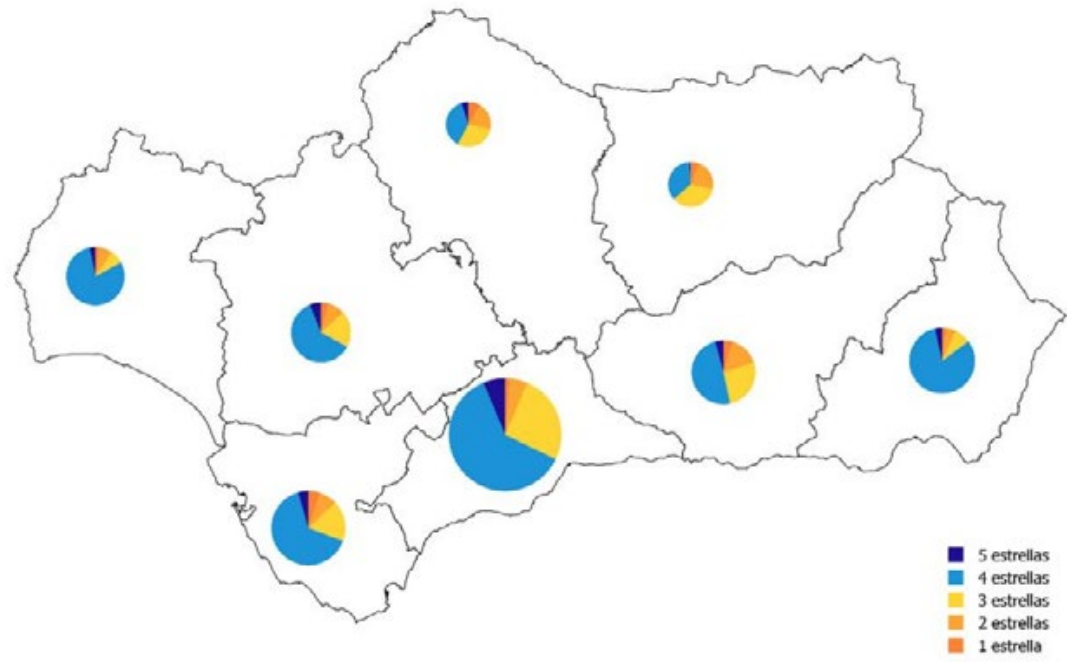

Fuente: Consejería de Turismo y Deporte, 2018. 


\section{REDES SOCIALES Y TURISMO ANDALUZ}

Las redes sociales online, como uno de grandes pilares del universo Web 2.0, pueden definirse como plataformas de Internet que permiten a los usuarios registrarse para generar un perfil, en el que plasmar datos e información personal o profesional, interactuar con el resto de los usuarios compartiendo información mediante mensajes, imágenes o videos, así como localizar a otros usuarios en función de las características publicadas en sus perfiles (INTECO, 2009).

En los últimos años no sólo ha aumentado el porcentaje de internautas activos en redes sociales (Gráfico 3) sino que se ha ampliado su frecuencia de utilización. Así, según el estudio de "Uso y actitudes de consumo de contenidos digitales" (ONTSI, 2017) el 40\% de la población española entre 16 y 74 años utiliza alguna plataforma social online todos los días, aumentando este porcentaje hasta el $52 \%$ si englobamos a todos aquellos que acceden a redes sociales al menos una vez por semana.

\section{Gráfico 3 \\ EVOLUCIÓN DE LA PENETRACIÓN DEL USO DE LAS REDES SOCIALES EN ESPAÑA (2009-2019)}

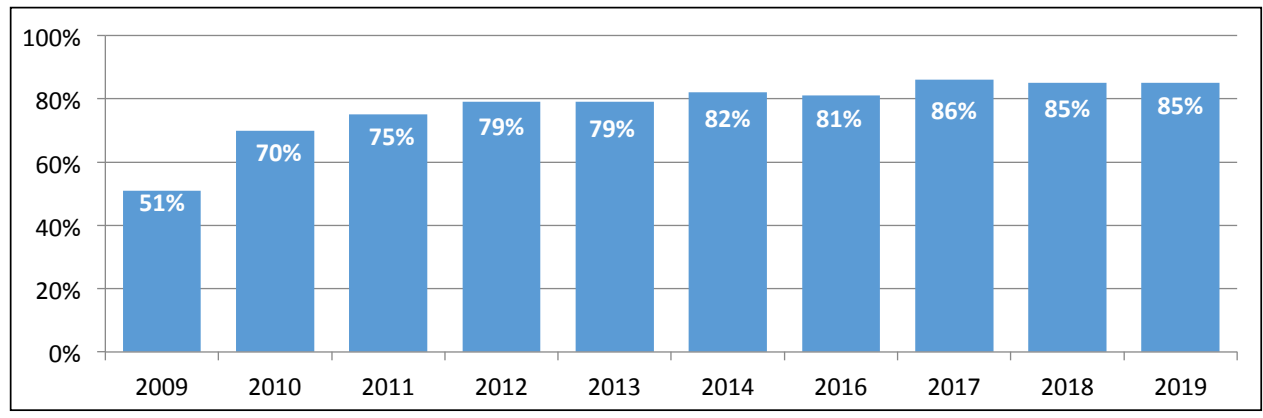

Fuente: IAB Spain, 2019.

Tomando como base la definición anterior, existe un gran número de redes sociales online con mayor o menor penetración de mercado y que se pueden encuadrar en alguno de estos dos grandes grupos: a) las redes sociales puras, que son las originariamente consideradas como tales y b) las plataformas de contenidos y microblogs que han ido incorporando la interacción con otros usuarios y la posibilidad de localizar y seguir a un determinado perfil (Tabla 1).

De ellas, las más utilizadas a nivel mundial, incluyendo España, son Facebook y YouTube (Gráfico 4). Por lo que respecta a las diferencias entre los usuarios españoles y mundiales, debemos destacar, especialmente, Google+. Aunque esta red alcanzó una cuota relevante en España en 2018, su declive mundial llevó a Google, el 8 de octubre de dicho año, a anunciar su cierre para los consumidores finales. 
Tabla 1

REDES SOCIALES

\begin{tabular}{|l|l|l|l|l|}
\hline Nombre & Año inicio & Origen & $\begin{array}{l}\text { Formato de intercambio } \\
\text { de información }\end{array}$ & Caracterización \\
\hline LinkedIn & 2003 & EE.UU. & Mensajes, fotos y videos & $\begin{array}{l}\text { Red social } \\
\text { (perfil profesional) }\end{array}$ \\
\hline Facebook & 2004 & EE.UU. & Mensajes, fotos y videos & $\begin{array}{l}\text { Red social } \\
\text { (perfil personal y profesional) }\end{array}$ \\
\hline Flickr & 2004 & EE.UU. & Fotos y videos & Plataforma de contenidos \\
\hline YouTube & 2005 & EE.UU. & Video & Plataforma de contenidos \\
\hline Twitter & 2006 & EE.UU. & Mensajes, fotos y videos & Microblog \\
\hline Pinterest & 2008 & EE.UU. & Fotos & Plataforma de contenidos \\
\hline Weibo & 2009 & China & Mensajes, fotos y videos & $\begin{array}{l}\text { Red social } \\
\text { (perfil personal y profesional) }\end{array}$ \\
\hline Instagram & 2010 & EE.UU. & Fotos y videos & Plataforma de contenidos \\
\hline Google + & 2011 & EE.UU. & Mensajes, fotos y videos & $\begin{array}{l}\text { Red social } \\
\text { (perfil personal y profesional) }\end{array}$ \\
\hline
\end{tabular}

Fuente: elaboración propia.

El sector turístico no es ajeno a esta popularidad de las redes sociales, viéndose afectado por el creciente impacto que han adquirido en el proceso de elección, planificación, desarrollo y postventa del servicio turístico. Concretamente, según el "Balance del Año Turístico en Andalucía 2016”, en el que se valoraron las fuentes de información a la hora de elegir destino y/o planificar el viaje a Andalucía (Consejería de Turismo y Deporte, 2017a.), el 40,5\% de los turistas se informó por medio de otros viajeros a través de alguna red social (Gráfico 5), mientras que el 29,5\% se apoyó en la opinión de amigos y conocidos en medios sociales, alcanzando la suma de ambos porcentajes un valor muy cercano al 67\% acumulado recogido por el Barómetro de TripAdvisor relativo al mismo año (TripAdvisor, 2016). Por lo que respecta al tiempo transcurrido durante el viaje, las actividades relacionadas con los medios sociales fueron las más utilizadas al usar Internet en sus dispositivos móviles (el 73,1\% publicó fotos y videos y el 61,6\% puso comentarios o recomendó lugares), aumentando estos porcentajes una vez finalizado el viaje hasta el $75 \%$ y $67,8 \%$, respectivamente. Además, Facebook se consolidó como la red social más usada para compartir contenidos del viaje, utilizándola el 80,2\% de los turistas, seguida por Instagram $(29,2 \%)$ y Twitter $(22,7 \%)$ (Consejería de Turismo y Deporte, 2017a). 
Gráfico 4

PORCENTAJE DE UTILIZACIÓN DE REDES SOCIALES EN RELACIÓN CON LOS USUARIOS DE INTERNET (ENERO 2018)

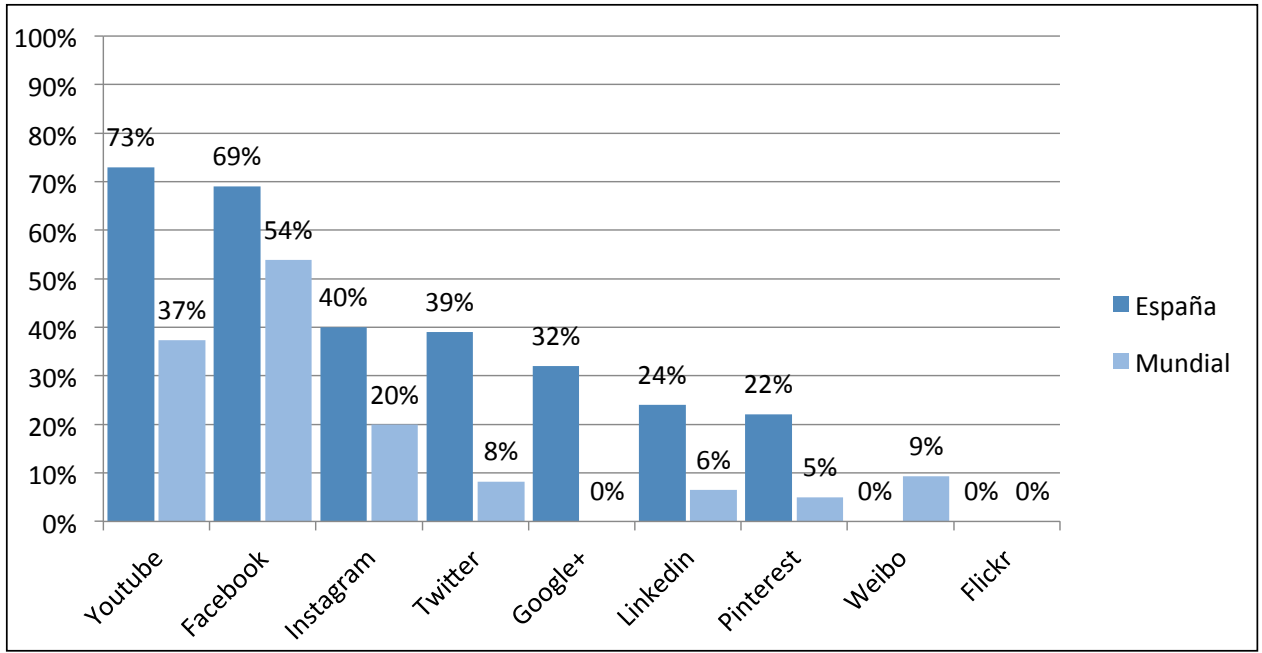

Fuente: Kemp, 2018.

Gráfico 5

FUENTES DE INFORMACIÓN A LA HORA DE ELEGIR DESTINO Y/O

PLANIFICAR EL VIAJE A ANDALUCÍA (\%)

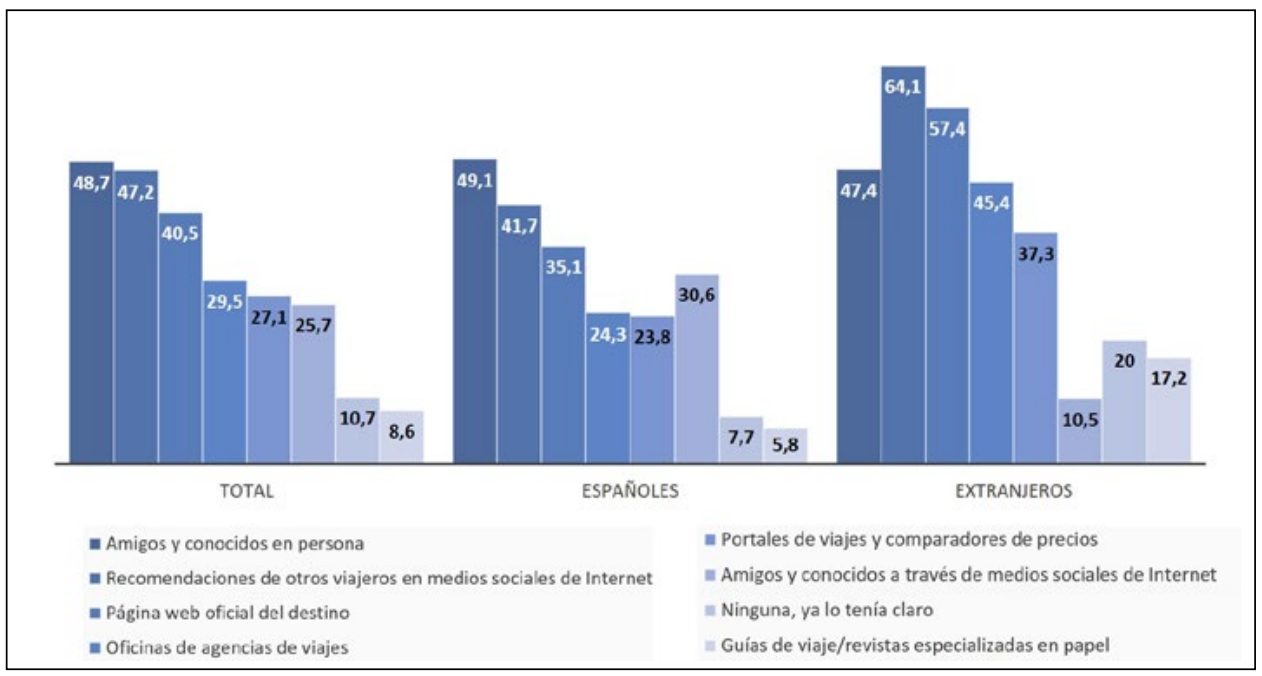

Fuente: Consejería de Turismo y Deporte, 2017a. 


\section{METODOLOGÍA}

\subsection{Diseño del estudio}

Se diseñó un estudio observacional transversal mediante la consulta de la página web oficial de los hoteles andaluces de 4 y 5 estrellas en activo, de todo el territorio andaluz. La recogida de información se realizó en el mes de enero de 2018 partiendo del listado facilitado por el Registro de Turismo de Andalucía (Consejería de Turismo y Deporte, 2017 b) y excluyendo aquellos establecimientos que, tras la búsqueda de su página web, se confirmó que no se mantenían activos, su número de estrellas no era correcto o no disponían de página web oficial.

Para cada uno de los hoteles resultantes se procedió a analizar las siguientes variables: 1) número de estrellas, 2) modalidad, 3) fecha de inicio de la actividad, 4) tamaño, 5) pertenencia a cadena hotelera, 6) número de redes sociales y 7) denominación de dichas redes. Para evaluar cada una de estas variables se establecieron diferentes categorías, las cuales aparecen reflejadas en la Tabla 2.

En relación a la modalidad hotelera, la Junta de Andalucía diferencia cuatro categorías: carretera, rural, ciudad y playa. Dado el bajo número de hoteles de carretera censados (tan solo estaban registrados 6 establecimientos de esta modalidad) se agruparon con los de categoría rural para poder obtener resultados estadísticamente representativos en nuestros estudios.

Por lo que respecta a la fecha de inicio de la actividad, se establecieron dos opciones: que el hotel fuera anterior o posterior a 2010, año en el que se potenció el uso de las redes sociales y comenzaron los primeros estudios de penetración de mercado en España. En lo referente al tamaño, se consideraron tres categorías: establecimiento pequeño, mediano y grande, según que el número de habitaciones fuera como máximo de 50, estuviera entre 50 y 300 o fuera mayor de 300 .

Con respecto a las redes sociales se estudió tanto el número como la denominación. El primer aspecto permitió clasificar a los establecimientos en cuatro grupos: hoteles con ninguna red, con un número escaso de redes (de 1 a 2), un valor medio (entre 3 y 4) o con alto número de redes (más de 4). Por lo que respecta a la denominación, se analizó cuáles de las siguientes 9 redes sociales aparecían en sus páginas web: Facebook, Flickr, Google+, LinkedIn, Pinterest, Twitter, YouTube y Weibo.

Tabla 2

VARIABLES ANALIZADAS

\begin{tabular}{|l|l|l|}
\hline Variables & Categorías & Origen de la información \\
\hline 1. Número de estrellas & $\begin{array}{l}4 \text { estrellas } \\
5 \text { estrellas }\end{array}$ & Registro de Turismo de Andalucía \\
\hline 2. Modalidad & $\begin{array}{l}\text { Carretera o rural } \\
\text { Ciudad } \\
\text { Playa }\end{array}$ & Registro de Turismo de Andalucía \\
\hline
\end{tabular}




\begin{tabular}{|l|l|l|}
\hline Variables & Categorías & Origen de la información \\
\hline $\begin{array}{l}\text { 3. Fecha de inicio de ac- } \\
\text { tividad }\end{array}$ & $\begin{array}{l}\text { Anterior a 2010 } \\
\text { A partir de 2010 }\end{array}$ & Registro de Turismo de Andalucía \\
\hline 4. Tamaño & $\begin{array}{l}\text { Pequeño (<50 habitaciones) } \\
\text { Mediano (50-300 habitaciones) } \\
\text { Grande (> 300 habitaciones) }\end{array}$ & Registro de Turismo de Andalucía \\
\hline $\begin{array}{c}\text { 5. Pertenencia a cadena } \\
\text { hotelera }\end{array}$ & $\begin{array}{l}\text { Independiente } \\
\text { Asociado a alguna cadena } \\
\text { hotelera }\end{array}$ & Página web oficial \\
\hline $\begin{array}{c}\text { 6. Número de redes so- } \\
\text { ciales }\end{array}$ & $\begin{array}{l}\text { Ninguna } \\
\text { Escaso (1-2 redes) } \\
\text { Medio (3-4 redes) } \\
\text { Alto (>4 redes) }\end{array}$ & Página web oficial \\
\hline $\begin{array}{c}\text { 7. Denominación de las } \\
\text { redes sociales }\end{array}$ & $\begin{array}{l}\text { Facebook, Flickr, Google+, } \\
\text { LinkedIn, Pinterest, Twitter, } \\
\text { YouTube, Weibo }\end{array}$ & Página web oficial \\
\hline
\end{tabular}

Fuente: elaboración propia.

Tras un análisis descriptivo basado en las frecuencias de las variables, se estudiaron posibles relaciones de dependencia entre las mismas, según el contraste de hipótesis con el estadístico de la Chi-Cuadrado, mediante el programa SPSS versión 20 (IBM Corp., Armonk, N.Y., USA). En concreto, se plantearon cinco hipótesis, que trataron de analizar si las variables número de estrellas, modalidad, antigüiedad, tamaño y pertenencia a una cadena pueden influir en el número y la elección las redes sociales.

\subsection{Hipótesis del estudio}

Las hipótesis planteadas fueron las siguientes:

Hipótesis 1: El número de estrellas del hotel determina el número y la elección de las redes sociales

Los requerimientos de tiempo y conocimientos de gestión de redes sociales pueden ser determinantes de su número, de manera que los hoteles de mayor categoría, con más recursos, pueden ser más propensos a tener más redes que los hoteles de menos estrellas. Además, las redes sociales elegidas por los hoteles de categoría superior podrían ser diferentes, al centrarse en un perfil de cliente distinto del que tienen los hoteles de cuatro estrellas.

En esta línea se encuentra el trabajo de Rodríguez y Sánchez (2015), quienes llegaron a la conclusión de que los hoteles de 3,4 y 5 estrellas tenían una presencia en Facebook y Twitter mayor que la de los establecimientos de menor categoría. Igualmente, Mellinas et al. (2014), concluyeron que los hoteles con mayor número de estrellas tienden a usar las redes sociales con mayor frecuencia y de forma más adecuada. 
Hipótesis 2: La modalidad del hotel es independiente del número y la elección de redes sociales

Hay estudios que reflejan diferentes grados de penetración de las redes sociales en función de la tipología de alojamiento turístico, según se trate de hoteles, apartamentos turísticos, campings o incluso alojamientos rurales, llegando a la conclusión de que los campings tenían una mayor presencia en Facebook, mientras que los hoteles de 3, 4 y 5 estrellas la tenían en Twitter (Rodríguez y Sánchez, 2015). Este estudio separaba los hoteles de modalidad rural del resto, incluyéndolos en el grupo de alojamientos rurales, con diferentes niveles de presencia en redes sociales. Sin embargo, si se tiene en consideración todas las modalidades hoteleras, a diferencia de otras características del establecimiento, no parece, a priori, ser determinante del número y la elección de redes sociales ofrecidas en la web del hotel.

Hipótesis 3: La antigüedad del hotel influye en el número y la elección de las redes sociales

Si bien en el estudio sobre hoteles balnearios realizado por Sánchez et al. (2014), los establecimientos más antiguos eran los que, curiosamente, mejor se habían adaptado a las redes sociales, con esta hipótesis nos planteamos que los establecimientos creados con posterioridad al auge de las redes sociales, al haber comenzado su actividad dentro de este entorno, habrían incorporado, desde sus inicios, de manera más natural, los medios sociales en su empresa. A diferencia de estos hoteles, los establecimientos con mayor antigüedad se encontrarían en proceso de incorporación de las redes sociales a sus páginas ya que habrían tenido que ir adaptándose a los cambios producidos tras la llegada de la Web 2.0.

Hipótesis 4: El tamaño de los hoteles incide sobre el número y la elección de las redes sociales

El contenido y las estrategias en las páginas web de los hoteles y, por tanto, la inclusión de las redes sociales en las mismas puede estar condicionado, entre otras variables, por el tamaño del hotel (Escobar-Rodríguez y Carvajal-Trujillo, 2013)

Bajo el planteamiento de que mayores recursos económicos permitirían una mejor gestión de las redes sociales, los hoteles de mayor tamaño utilizarían más intensamente las nuevas tecnologías (Buhalis y Law, 2008; Ruiz et al., 2012; Ortiz y González, 2014). Por ello nos planteamos que los hoteles más pequeños, normalmente familiares y con menores recursos, podrían tener más dificultad para manejar estas plataformas, lo que influiría en el número y la elección de éstas.

En esta línea se encuentra el trabajo de Ortiz y González (2014), relativo a hoteles de tres estrellas de Madrid Capital, llegando a la conclusión de que, sobre todo, los hoteles de menor tamaño, no incluían en sus planes de marketing la gestión de las redes sociales.

Hipótesis 5: La pertenencia a una cadena hotelera es determinante del número y la elección de las redes sociales

Existen estudios que justifican esta hipótesis en Andalucía (Moral et al., 2014; Cañero et al., 2015), en los que se concluye que la disponibilidad de redes sociales es más alta en 
los establecimientos pertenecientes a cadenas hoteleras. Además, la importancia otorgada a las redes sociales también puede estar relacionada con la pertenencia del hotel a una cadena, ya que estos establecimientos tienen un desarrollo superior de las plataformas sociales que los hoteles independientes, utilizándolas como canales de ventas y controlando los resultados de tales acciones (Mellinas et al., 2014; Cascales et al., 2017) Por otro lado, en un estudio realizado en 2011 en Estados Unidos, el 94\% de los hoteles pertenecientes a cadenas incluían al menos un tipo de red social en su web oficial (Aluri et al., 2016). Esto podría deberse a que los hoteles pertenecientes a una cadena pueden verse influenciados por políticas corporativas que pueden afectar al número y la elección de las redes sociales utilizadas.

En este sentido, hay estudios que muestran "una relación entre las valoraciones de las diferentes redes sociales por parte de los hoteles en función de si pertenecen a una cadena o si son hoteles independientes, salvo en el caso de Facebook y Twitter donde no hay relación entre la importancia otorgada a las redes sociales y si el hotel pertenece a una cadena o es independiente" (Cascales et al., 2017).

\section{RESULTADOS}

El análisis del Registro de Turismo de Andalucía proporcionó 522 establecimientos activos en 2017. Tras la revisión de sus páginas web oficiales, encontramos 503 establecimientos que proporcionaban datos analizables. La búsqueda de las webs nos mostró que estaban realmente en funcionamiento 507 establecimientos de 4 y 5 estrellas en Andalucía, de los cuales 4 no disponían de página web oficial.

\subsection{Caracterización de los hoteles andaluces de 4 y 5 estrellas}

Los resultados obtenidos tras el análisis de las variables aparecen reflejados en la Tabla 3. La mayoría de hoteles eran de 4 estrellas $(90,26 \%)$ y estaban localizados en la ciudad $(48,11 \%)$ o en la playa $(40,95 \%)$. Tan solo un $12,92 \%$ inició su actividad después del año 2010. Por lo que respecta al tamaño, el $62 \%$ podían considerarse hoteles medianos, el $25 \%$ grandes y casi el $13 \%$ pequeños, perteneciendo la mayoría de ellos a alguna cadena (69\%).

En relación con las redes sociales, se observó que solo el 12,5\% de los establecimientos no disponía de ninguna en su página web. La mayoría de los hoteles presentaba entre 3 y $4(39,76 \%)$ o más de $4(32,60 \%)$ (Gráfico 6), destacando especialmente Facebook, Twitter y Google+.

De modo detallado, el número de redes sociales presentes en los hoteles analizados se recoge en el Gráfico 6. 
Tabla 3

RESULTADOS DEL ANÁLISIS DESCRIPTIVO

\begin{tabular}{|c|c|c|c|}
\hline Variables & Categorías & \begin{tabular}{|l|} 
Número \\
de hoteles
\end{tabular} & \begin{tabular}{|l|} 
Porcentaje \\
de hoteles \\
\end{tabular} \\
\hline \multirow{2}{*}{ 1. Número de estrellas } & 4 estrellas & 454 & $90,26 \%$ \\
\hline & 5 estrellas & 49 & $9,74 \%$ \\
\hline \multirow{3}{*}{ 2. Modalidad } & Carretera o rural & 55 & $10,93 \%$ \\
\hline & Ciudad & 242 & $48,11 \%$ \\
\hline & Playa & 206 & $40,95 \%$ \\
\hline \multirow{2}{*}{ 3. Fecha de inicio de actividad } & Anterior a 2010 & 438 & $87,08 \%$ \\
\hline & A partir de 2010 & 65 & $12,92 \%$ \\
\hline \multirow{3}{*}{ 4. Tamaño } & Pequeño (< 50 habitaciones) & 129 & $25,65 \%$ \\
\hline & Mediano (50-300 habitaciones) & 312 & $62,03 \%$ \\
\hline & Grande (> 300 habitaciones) & 62 & $12,33 \%$ \\
\hline \multirow{2}{*}{$\begin{array}{l}\text { 5. Pertenencia a cadena } \\
\text { hotelera }\end{array}$} & Independiente & 156 & $31,01 \%$ \\
\hline & Asociado a alguna cadena hotelera & 347 & $68,99 \%$ \\
\hline \multirow{4}{*}{ 6. Número de redes sociales } & Ninguna & 63 & $12,52 \%$ \\
\hline & Escaso (1-2 redes) & 76 & $15,11 \%$ \\
\hline & Medio (4 redes) & 200 & $39,76 \%$ \\
\hline & Alto (> 4 redes) & 164 & $32,60 \%$ \\
\hline \multirow{8}{*}{$\begin{array}{l}\text { 7. Denominación de las } \\
\text { redes sociales (por orden de } \\
\text { relevancia) }\end{array}$} & Facebook & 430 & $85,49 \%$ \\
\hline & Twitter & 373 & $74,16 \%$ \\
\hline & Google+ & 269 & $53,48 \%$ \\
\hline & YouTube & 231 & $45,92 \%$ \\
\hline & Instagram & 194 & $38,57 \%$ \\
\hline & Pinterest & 126 & $25,05 \%$ \\
\hline & \begin{tabular}{|l} 
LinkedIn \\
\end{tabular} & 80 & $15,90 \%$ \\
\hline & Flickr & 65 & $12,92 \%$ \\
\hline
\end{tabular}

Fuente: elaboración propia. 


\section{Gráfico 6}

NÚMERO DE REDES SOCIALES OFRECIDAS POR LOS HOTELES ANDALUCES EN SUS PÁGINAS WEB OFICIALES

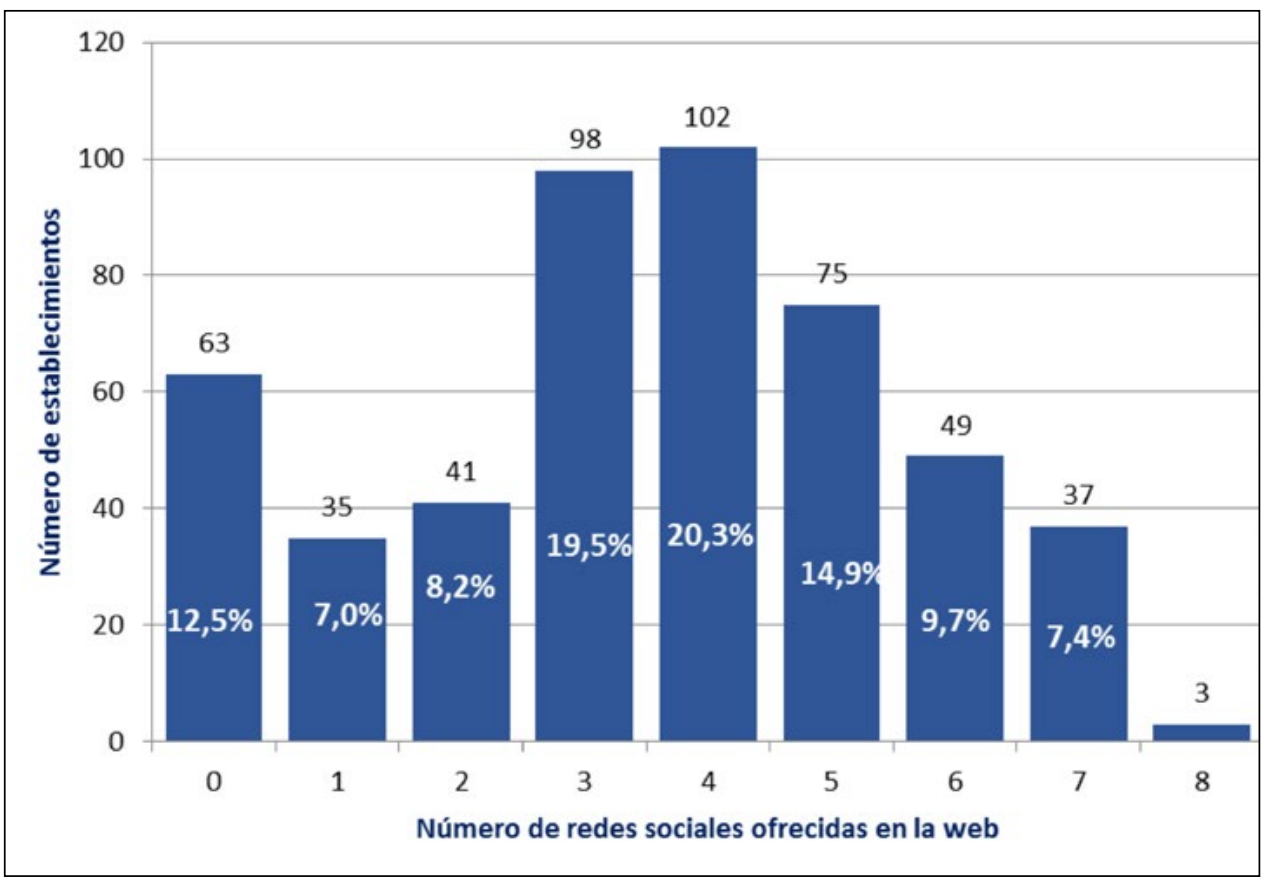

Fuente: elaboración propia.

\subsection{Relaciones entre variables analizadas: contraste de hipótesis}

De las 9 redes sociales consideradas, en el análisis de las relaciones entre variables se excluyó Weibo debido a que sólo estaba presente en 3 hoteles y, por tanto, los resultados no eran representativos.

Teniendo esto en cuenta, los resultados obtenidos con respecto a las hipótesis planteadas fueron las indicadas en la Tabla 4. 


\section{Tabla 4 \\ RESULTADOS DEL CONTRASTE DE HIPÓTESIS CON EL ESTADÍSTICO DE LA CHI-CUADRADO $(\mathbf{P}<0,05)$}

\begin{tabular}{|c|c|c|c|c|c|c|c|c|c|c|}
\hline & \multirow{2}{*}{\multicolumn{2}{|c|}{$\begin{array}{c}\text { Hipótesis } 1 \\
\text { Estrellas }\end{array}$}} & \multirow{2}{*}{\multicolumn{2}{|c|}{$\begin{array}{l}\text { Hipótesis } 2 \\
\text { Modalidad }\end{array}$}} & \multirow{2}{*}{\multicolumn{2}{|c|}{$\begin{array}{l}\text { Hipótesis } 3 \\
\text { Antigüiedad }\end{array}$}} & \multirow{2}{*}{\multicolumn{2}{|c|}{$\begin{array}{c}\text { Hipótesis } 4 \\
\text { Tamaño }\end{array}$}} & \multirow{2}{*}{\multicolumn{2}{|c|}{$\begin{array}{c}\text { Hipótesis } 5 \\
\begin{array}{c}\text { Pertenencia a } \\
\text { cadena }\end{array}\end{array}$}} \\
\hline & & & & & & & & & & \\
\hline & Valor & Sig. & Valor & Sig. & Valor & Sig. & Valor & Sig. & Valor & Sig. \\
\hline $\begin{array}{l}\text { Número } \\
\text { de redes } \\
\text { sociales }\end{array}$ & 2 & 0,001 & $48, \varepsilon$ & 0,000 & 3,447 & 0,328 & 6 & 0,000 & 53,786 & 0,000 \\
\hline Facebook & 4,761 & 0,029 & 16,9 & 0,000 & 0,938 & 0,333 & 5,359 & 0,069 & 0,138 & 0,710 \\
\hline Flickr & 1,43 & 0,232 & 14,194 & 0,001 & 1,815 & 0,178 & 12,259 & 0,002 & 3,132 & 0,070 \\
\hline Google+ & 501 & 0,001 & 11 & 0,0 & 4,2 & 0,0 & 10,8 & 0,0 & 10,079 & 0,001 \\
\hline Instagram & 16,38 & 0,000 & 11,346 & 0,003 & 1,152 & 0,283 & 20,715 & 0,000 & 12,944 & 0,000 \\
\hline LinkedIn & 1,738 & 0,187 & 11,85 & 0,003 & 0,936 & 0,333 & 1,58 & 0,454 & 17,368 & 0,000 \\
\hline Pinterest & 5,448 & 0,020 & 9,117 & 0,010 & 0,048 & 0,826 & 4,71 & 0,095 & 14,434 & 0,000 \\
\hline YouTube & 1,113 & 0,291 & 22,92 & 0,000 & 1,055 & 0,304 & 20,895 & 0,000 & 22,721 & 0,000 \\
\hline Twitter & 5,23 & 0,022 & 17,677 & 0,000 & 3,544 & 0,060 & 20,976 & 0,000 & 18,782 & 0,000 \\
\hline
\end{tabular}

Fuente: elaboración propia.

Hipótesis 1: El número de estrellas del hotel determina el número y la elección de las redes sociales

El estadístico de la Chi-cuadrado $\left(\chi^{2}=16,012, \mathrm{P}=0,001, \mathrm{P}<0,05\right)$ demostró que existía una relación entre las estrellas de un hotel y el número de redes sociales. Concretamente, los establecimientos de 5 estrellas tenían de media un número superior.

En el análisis individual se apreció que, excepto para el caso de Flickr, YouTube y LinkedIn, existía una dependencia entre el número de estrellas y la elección de las plataformas sociales que los hoteles mostraban en sus páginas web.

Hipótesis 2: La modalidad del hotel es independiente del número y la elección de redes sociales

Se demostró que las variables modalidad y número de redes estaban relacionadas $\left(\chi^{2}\right.$ $=48,847, \mathrm{P}=0,000, \mathrm{P}<0,05)$. Concretamente, se observó que los hoteles de playa tenían un mayor número de redes sociales que el resto, con una media superior a 4.

Estudiando de manera individual cada una de las redes se observó además que, dependiendo de la modalidad, los hoteles elegían una plataforma u otra. Así, los hoteles de carretera o rurales se decantaban por las redes más habituales como Facebook, Twitter y Google+, mientras que los hoteles de ciudad y los de playa apostaban, además, por YouTube e Instagram, respectivamente. 
Hipótesis 3: La antigüedad del hotel influye en el número y la elección de las redes sociales

Los resultados mostraron que no existían diferencias significativas en el número de redes sociales entre los hoteles creados antes de la era de la Web 2.0 y los establecimientos más actuales $\left(\chi^{2}=3,447, \mathrm{P}=0,328\right)$.

En cuanto a la elección de redes tampoco se apreciaron diferencias, a excepción de Google+ que tenía una mayor presencia en los hoteles con fecha de inicio de actividad posterior a 2010 .

Hipótesis 4: El tamaño de los hoteles incide sobre el número y la elección de las redes sociales

El contraste de hipótesis mostró que había diferencias significativas en el número de redes sociales según el tamaño de los establecimientos $\left(\chi^{2}=55,596, \mathrm{P}=0,000, \mathrm{P}<0,05\right)$, ya que los hoteles más grandes tenían un mayor número ( 4 de media frente a las 2 de los hoteles pequeños).

Por lo que respecta al análisis individualizado de las redes se evidenciaron diferencias significativas en la mayoría de ellas, a excepción de Facebook, Pinterest y LinkedIn que estaban presentes a un nivel similar, independientemente del tamaño del hotel.

Hipótesis 5: La pertenencia a una cadena hotelera es determinante del número y la elección de las redes sociales

El estudio de la Chi-cuadrado mostró que existía una relación entre el hecho de pertenecer a una cadena hotelera y el número de redes sociales presentes en las webs $\left(\chi^{2}=\right.$ $53,786, \mathrm{P}=0,000, \mathrm{P}<0,05)$. Además, todos los establecimientos integrados en una misma cadena mostraban las mismas redes.

Si se analizaba cada red por separado, en la mayoría de ellas se podía llegar a la conclusión de que existía una relación de dependencia con el hecho de que el hotel perteneciera a una cadena, salvo en el caso de Facebook y Flickr, para las cuales los niveles de significación de la Chi-cuadrado indicaban lo contrario ( $\mathrm{P}=0,710$ y $\mathrm{P}=0,070$, respectivamente).

\section{CONCLUSIONES}

La importancia de las redes sociales para potenciar la imagen de marca y su uso como fuente de información en la planificación del turismo, se ha recogido en diferentes informes (Consejería de Turismo y Deporte, 2017a; IAB Spain, 2019). Sin embargo, a pesar de su relevancia, la investigación en este ámbito necesita ser ampliada en España, dado que los estudios que abordan cómo se han adaptado los hoteles españoles a las redes sociales presentan un alcance limitado. Así, o sólo analizan 4 redes sociales, o incorporan un número reducido de variables, o no realizan un análisis estadístico que permita conocer la relación entre ellas.

Los resultados de nuestro estudio evidencian dos importantes aspectos. En primer lugar, el esfuerzo de los hoteles andaluces de 4 y 5 estrellas por incorporarse al Turismo 2.0 , ya que una amplia mayoría $(87,5 \%)$ disponen de redes sociales en sus páginas web y, por otra parte, su apuesta por la diversificación en las redes para cubrir un gran número de 
perfiles distintos de clientes potenciales. En este sentido, debemos destacar que el 72,36\% de los hoteles cuentan con más de 3 de redes en sus webs, situándose el porcentaje más alto en el intervalo de entre 3 y 4 . Este aspecto es especialmente importante, ya que hoy en día la utilización de las redes sociales y, especialmente, la gestión de las opiniones online constituye una valiosa herramienta de marketing en el sector hotelero.

En lo referente a los hoteles que no incluyen redes en sus páginas web, un total de 63 establecimientos, merece la pena destacar que el $70 \%$ de ellos pertenece a una cadena hotelera. Este hecho puede estar originado por una política común de gestión, que puede incluso implicar una plataforma social única para todo el grupo, con las correspondientes economías de escala (Cañero et al., 2015). Por otra parte, y con respecto al 30\% de hoteles restante, es decir los establecimientos independientes que no disponen de redes sociales en sus páginas web, podría ser muy útil que las incorporaran y utilizaran como canales de ventas (Mellinas et al., 2014; Martín y Arteaga, 2016; Cascales et al., 2017). Además, y dado que estas plataformas actúan como una fuente básica de información en el proceso de planificación y elección del destino turístico, su utilización facilitaría la adecuación de los establecimientos a los cambios en los patrones del consumo (Cox et al., 2009). Por todo ello, pensamos que sobre dichos hoteles debería recaer gran parte de los esfuerzos institucionales con el objetivo de incorporar las redes sociales en sus webs, en aras de una mejora en las ventas, en la relación con sus clientes y en una gestión más adecuada de su reputación online.

En relación con el número de redes sociales, los establecimientos que cuentan con un mayor número son los hoteles de 5 estrellas, los de mayor tamaño y los de playa, destacando, especialmente, Facebook, Twitter y Google+, sobre todo en los hoteles de mayor categoría. El hecho de que Facebook sea la red social con mayor presencia también ha sido recogido en estudios previos (Martínez et al., 2013; Mellinas et al., 2014; Moral et al., 2014; Cascales et al. 2015; Martín y Arteaga, 2016), sin embargo, no tenemos constancia de que existan trabajos sobre otras como Google+. Esto puede ser debido a que la mayoría de los estudios existentes han trabajado con datos anteriores a 2013 o que, quizás, hasta ese momento dicha red social no había adquirido el nivel de penetración que alcanzó en España en 2018, un 32\% (Kemp, 2018), por lo que no resultaba relevante su análisis.

En términos generales, hemos evidenciado que la jerarquía de redes sociales no depende del número de estrellas del establecimiento, salvo la distorsión provocada por YouTube e Instagram que en los hoteles de 4 estrellas ocupan la posición cuarta y quinta respectivamente, mientras que en los de 5 estrellas las intercambian. Esto puede ser debido a la importancia que tiene la imagen en los establecimientos de categoría superior, donde predominan los hoteles con encanto, lujo y exclusividad.

En lo referente a las hipótesis planteadas, han quedado patentes las relaciones existentes entre el número de redes sociales presentes en las páginas web de los hoteles con: 1) el número de estrellas, 2) la modalidad, 3) el tamaño y 4) la pertenencia a una cadena. Con respecto al vínculo entre el número de redes sociales y la modalidad, destaca el hecho de los hoteles de playa sean los que ofrecen un mayor número de éstas, lo cual puede ser debido a un intento de llegar a una mayor variedad de clientes potenciales (familias, jubilados, congresos, etc.) que hagan frente a la alta estacionalidad de la demanda de este tipo de establecimientos. Por su parte, la relación entre el número de redes y la pertenencia 
a una cadena, se ha evidenciado en estudios previos (Martínez et al., 2012 y 2013; Cascales et al., 2017; Mellinas et al., 2016) y puede ser debida a políticas corporativas y a la búsqueda de economías de escala, utilizándose, en algunos casos, una única plataforma social del grupo para todos los hoteles integrantes del mismo. Además, esta relación puede estar conectada con el tamaño del establecimiento. De esta forma, los hoteles de menor tamaño tienen menos probabilidad de pertenecer a una cadena, lo cual, unido a una menor disponibilidad de recursos, conllevaría a ofertar un menor número de redes sociales en sus páginas web.

Somos conscientes de que las conclusiones de este estudio no pueden generalizarse, debido a que la investigación se ha centrado en los hoteles de 4 y 5 estrellas de Andalucía, caracterizados por un alto índice de asociación a cadenas hoteleras, lo cual puede incidir sobre los recursos disponibles para la gestión de las redes sociales. No perdemos de vista que las redes pueden ser especialmente útiles para los hoteles de categorías inferiores ya que podrían ayudarles a mejorar su comunicación, ventas y reputación a un coste inferior al de los canales tradicionales. Por este motivo, dedicaremos futuras investigaciones a analizar su presencia en dichos establecimientos, así como ampliar el ámbito geográfico para analizar posibles paralelismos y obtener unas conclusiones más generales.

Igualmente, resultaría interesante analizar, no solo qué redes sociales utilizan los hoteles, sino también cómo las están gestionando y el posible efecto, que tiene o puede tener, una gestión eficiente de las mismas en los resultados empresariales.

\section{REFERENCIAS}

ALURI, A., SLEVITCH, L. y LARZELERE, R. (2016): «The Influence of Embedded Social Media Channels on Travelers' Gratifications, Satisfaction, and Purchase Intentions», Cornell Hospitality Quarterly, vol. 57 (3), pp. 250-267.

AYEH, J., LEUNG, D., AU, N. y LAW, R. (2012): «Perceptions and Strategies of Hospitality and Tourism Practitioners on Social Media: An Exploratory Study», en Fuchs, M., Ricci, F., Cantoni, L. (Eds.) Information and Communication Technologies in Tourism. Vienna, Springer, pp. 1-12.

BUHALIS, D. y LAW, R. (2008): «Progress in information technology and tourism management: 20 years on and 10 years after the Internet-the state of eTourism research», Tourism Management, vol. 29 (4), pp. 609-623.

CAÑERO MORALES, P., ORGAZ ARGÜERA, F. y MORAL CUADRA, S. (2015): «Análisis de las variables que influyen en la reputación online de las empresas turísticas. El caso de los hoteles de Córdoba y Granada», Gran Tour, n 11, pp. 103-120.

CASCALES, G., FUENTES, L y ESTEBAN, J. DE. (2017): «La interacción de los hoteles con las redes sociales: un análisis de los hoteles de cuatro estrellas de la Comunidad de Madrid (España)», Cuadernos de Turismo, no 39, pp. 131-148.

CONSEJERÍA DE TURISMO Y DEPORTE (2017a): BATA - Balance del Año Turístico en Andalucía 2016. Junta de Andalucía. Disponible en https://www.turismoandaluz. com/ estadisticas/sites/default/files/bata_2016.pdf.

CONSEJERÍA DE TURISMO Y DEPORTE (2017b): RTA - Registro de Turismo de Andalucía. Junta de Andalucía. 
CONSEJERÍA DE TURISMO Y DEPORTE (2018): BATA - Balance del Año Turístico en Andalucía 2017. Junta de Andalucía. Disponible en https://www.turismoandaluz. com/ estadisticas/sites/default/files/bata_2017.pdf.

COX, C., BURGESS, S., SELLITTO, C. y BUULTJENS, J. (2009): «The role of usergenerated content in tourists' travel planning behavior», Journal of Hospitality Marketing and Management, vol. 18 (8), pp. 743-764.

ESCOBAR-RODRÍGUEZ, T. y CARVAJAL-TRUJILLO, E. (2013): «An evaluation of Spanish hotel websites: Informational vs. relational strategies», International Journal of Hospitality Management, vol. 33 (1), pp. 228-239.

FOTIS, J., BUHALIS, D. y ROSSIDES, N. (2012): «Social media use and impact during the holiday travel planning process», en Fuchs, M., Ricci, F. y Cantoni, L. (Eds) Information and Communication Technologies in Tourism. Vienna, Springer, pp. 13-24.

IAB SPAIN (2019): Estudio anual Redes sociales 2019. Disponible en https://iabspain.es/ wp-content/uploads/estudio-anual-redes-sociales-iab-spain-2019_vreducida.pdf

INTECO -Instituto Nacional de Tecnologías de la Comunicación- (2009): Estudio sobre la privacidad de los datos personales y la seguridad de la información en las redes sociales online. Disponible en: https://www.csirtcv.gva.es/sites/all/files/downloads/ estudio_intecoaepd_privacidad_redes_sociales.pdf.

KEMP, S. (2018): Digital in 2018 in Southern Europe: Essential Insights into Internet, Social Media, Mobile, and E-Commerce Use Across the Region. Disponible en https:// digitalreport.wearesocial.com/download.

KIETZMANN, J.H., HERMKENS, K., MCCARTHY, I.P. y SILVESTRE, B.S. (2011): «Social Media? Get Serious! Understanding the functional building blocks of social media», Business Horizons, vol. 54, (1), pp. 241-251.

LEUNG, D., LAW, R., VAN HOOF, H. y BUHALIS, D. (2013): «Social media in tourism and hospitality: a literature review», Journal of Travel \& Tourism Marketing, vol. 30 (1-2), pp. 3-22.

MARTÍN RIVERO, R. y ARTEAGA MARTÍN, M. (2016): «La Presencia En La Web Los Establecimientos Hoteleros de La Isla de Tenerife», Papers de Turisme, $\mathrm{n}^{\circ}$ 59, pp. 36-61.

MARTÍNEZ, S.M., BERNAL, J.J. y MELLINAS, J.P. (2012): «Los hoteles de la Región de Murcia ante las redes sociales y la reputación online», Revista de Análisis Turístico, $\mathrm{n}^{\mathrm{o}} 13$, pp. 1-10.

MARTÍNEZ, S.M., BERNAL, J.J. y MELLINAS, J.P. (2013): «Análisis del nivel de presencia de los establecimientos hoteleros de la región de Murcia en la Web 2.0», Cuadernos de Turismo, $\mathrm{n}^{\circ}$ 31, pp. 245-261.

MELLINAS, J.P., MARTÍNEZ, S. M. y BERNAL, J.J. (2014): «Uso de las redes sociales en los hoteles de la costa española», Investigaciones Turísticas, $\mathrm{n}^{\circ}$ 7, pp. 87-101.

MELLINAS, J.P., MARTÍNEZ, S.M. y BERNAL, J.J. (2016): «El uso de redes sociales por los hoteles como indicativo de gestión eficiente», Tourism \& Management Studies, vol. 12 (2), pp. 78-83.

MORAL CUADRA, S., CAÑERO MORALES, P. y ORGAZ ARGÜERA, F. (2014): «Reputación online, redes sociales y turismo. Un estudio de caso en Andalucía», Revista de Investigación en Turismo y Desarrollo Local, vol. 7 (16), pp. 1-17. 
ONTSI -Observatorio Nacional de las Telecomunicaciones y de la SI- (2017): Estudio de uso y actitudes de consumo de contenidos digitales. Disponible en http://www.ontsi. red.es/ontsi/sites/ontsi/files/Uso y actitudes de consumo de contenidos digitales. Julio 2017.pdf.

ORTIZ GARCÍA, L. y GONZÁLEZ SÁNCHEZ, R. (2014): «Las redes sociales como herramienta de mejora de la experiencia turística: una aplicación al sector hotelero», Revista Iberoamericana de Turismo, vol. 4 (1), pp. 16-34.

PHELAN K. V., CHEN, H.T. y HANEY, M. (2013): “"Like” and "Check-in": how hotels utilize Facebook as an effective marketing tool», Journal of hospitality and Tourism Technology, vol. 4 (2), pp. 134-154.

RODRÍGUEZ RANGEL, C. y SÁNCHEZ RIVERO, M. (2015): «Presencia de alojamientos extremeños en redes sociales», en VIII Jornadas de Investigación en Turismo, pp. 273-294.

RUIZ MOLINA, M.E., GIL SAURA, I. y MOLINER VELÁZQUEZ, B. (2012): «Tecnologías de la información en el sector hotelero y sus implicaciones en las relaciones empresa-cliente», Revista de Análisis Turístico, no 13, pp. 11-26.

SÁNCHEZ AMBOAGE, E., RODRÍGUEZ FERNÁNDEZ, M. M., MARTÍNEZ FERNÁNDEZ, V. A., RODRÍGUEZ VÁZQUEZ, C. y ÁLVAREZ DE LA TORRE, J. (2014): «El uso de los medios sociales Facebook y Twitter en los balnearios de Galicia», en XVIII Congreso AECIT, pp. 1-12.

SÁNCHEZ-FRANCO, M.J. y RONDAN-CATALUÑA, R.J. (2010): «Virtual travel communities and customer loyalty: Customer purchase involvement and web site design», Electronic Commerce Research and Applications, vol. 9 (2), pp.171-182.

SIGALA, M., GRETZEL, U. y CHRISTOU, E. (2012): Social Media in Travel, Tourism and Hospitality: Theory, Practice and Cases. Ashgate Publishing, Farnham, Surrey, Burlington, VT.

TRIPADVISOR (2016): Tripbarometer 2016: Traveler Trends \& Motivations Global Findings. Disponible en https://www.tripadvisor.es/ TripAdvisorInsights/tripbarometer.

TRIPADVISOR (2018): Tripbarometer 2017/2018: Global Report. Disponible en https:// www.tripadvisor.es/ TripAdvisorInsights/tripbarometer.

VERMA, R., STOCK, D. y MCCARTHY, L. (2012): «Customer preferences for online, social media, and mobile innovations in the hospitality industry», Cornell Hospitality Quarterly, vol. 53 (3), pp. 183-186.

ZEHRER, A., CROTTS, J. y MAGNINI, V. (2011): «The perceived usefulness of blog postings: An extension of the expectancy-disconfirmation paradigm», Tourism Management, vol. 32 (1), pp. 106-113.

ZENG, B. (2013): «Social media in tourism», Journal of Tourism and Hospitality, vol. 2 (1), pp. 1-2. 
